Distribution Catcgory:

Mathematics and Computer

Science (UC-405)

ANL-89/42

ANL- $-89 / 42$

DE90 006462

\author{
ARGONNE NATIONAL LABORATORY \\ 9700 South Cass Avenue \\ Argonne, IL 60439-4801
}

\title{
AN AUTOMATED PROCEDURE FOR \\ COVARIATION-BASED DETECTION OF RNA STRUCTUIRE
}

by

S. Winker, R. Overbeek, C.R.Woese," G.J.Olsen," and N. Pfuger ${ }^{\dagger}$

Mathematics and Computer Science Division

December 1989

-Address: Dept. of Molecular Biology, University of Illinois, Urbana, IL 61801.

${ }^{\dagger}$ Present address: Dept. of Computer Science, Texas A\&M University, College Station, TX 77840.

This work was supported in part by the Applied Mathematical Sciences subprogram of the Office of Energy Research. U.S. Department of Energy, under Contract W-31-109-Eng-38, and in part by C. R. Woese's NASA Grant NSG7044. 


\section{A major purpose of the Techni-}

cal Information Center is to provide the broadest dissemination possible of information contained in DOE's Research and Development Reports to business, industry, the academic community, and federal, state and local governments.

\section{Although a small portion of this} report is not reproducible, it is being made available to expedite the availability of information on the research discussed herein.

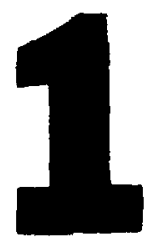

We present the validation of the automated procedure in four subsections. In the first subsection, we stress the need for accurate detection, especially when considering tertiary and quatemary structure. In the second subsection, we present the validation of a covariance-based method for detection of secondary structure, and compare the performance of this method with that of other published methods. In the third subsection, we present the validation of automated covariance analysis in the context of a phylogenctic tree. Finally, in the fourth subsection, we presert the validation of automated covariance analysis in the detection of teriary structure and generalized covariation. Note that validation of search methods for quaternary structure (bonding between the $16 \mathrm{~S}$ and $23 \mathrm{~S}$ RNA molecules) must be based on the corresponding valida- 


\section{Contents}

Abstract

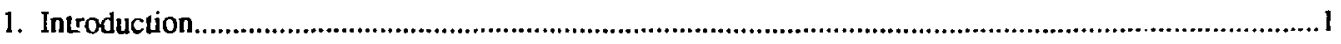

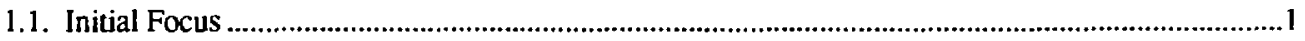

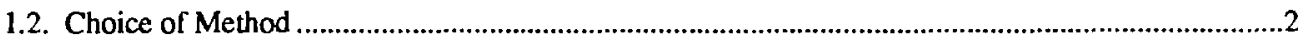

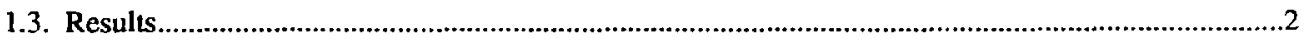

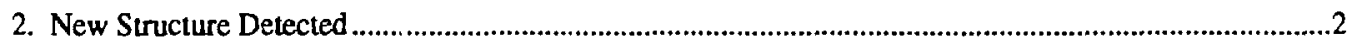

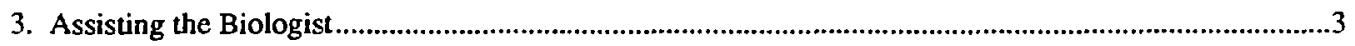

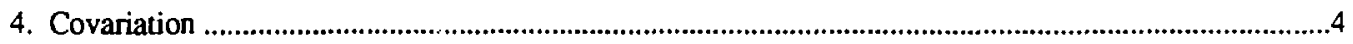

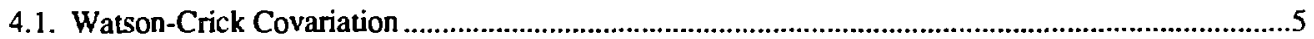

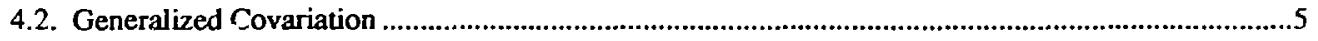

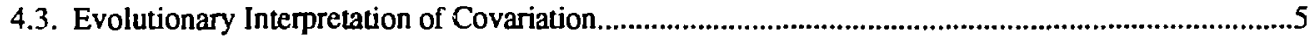

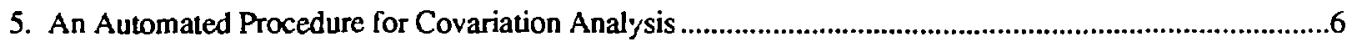

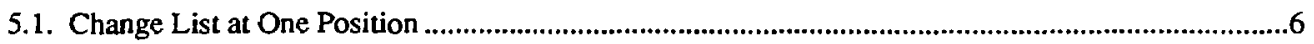

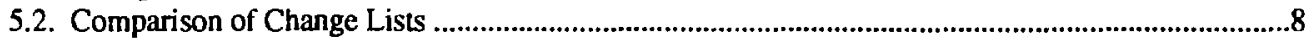

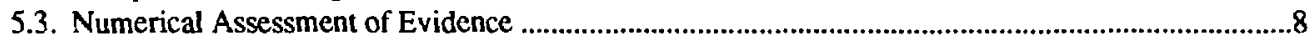

5.4. Example of Numerical Assessment of Evidence ..................................................................................11

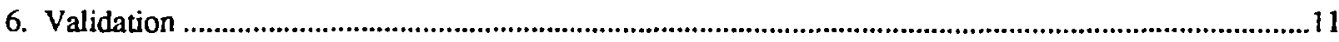

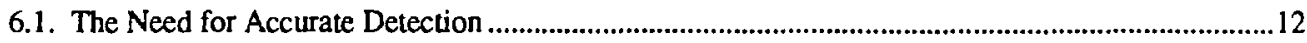

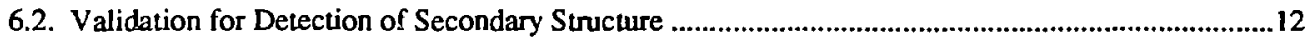

6.3. Validation for Covariation in a Phylogenetic Tree .............................................................................13

6.4. Validation for Tertiary Structure and Generalized Covariance ........................................................13

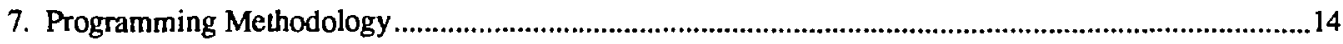

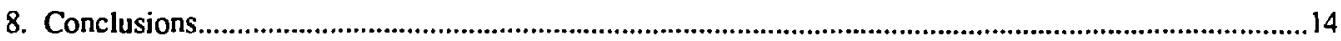

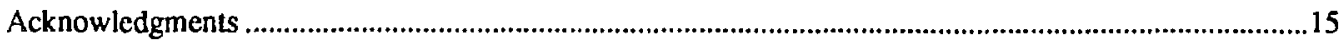

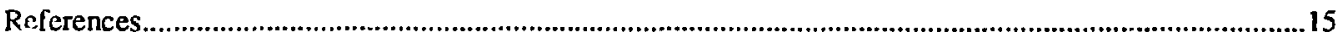




\title{
An Automated Procedure for Covariation-Based Detection of RNA Structure
}

by

S. Winker, R. Overbeek, C. R. Woese, G. J. Olsen, and N. Pfluger

\begin{abstract}
This paper summarizes our investigations into the compulational detection of secondary and tertiary structure of ribosomal RNA. We have developed a new automated procedure that not only identifies potential bondings of secondary and tertiary structure, but also provides the covariation evidence that supports the proposed bondings, and any counterevidence that can be detected in the known sequences. A small number of previously unknown bondings have been detected in individual RNA molecules (16S rRNA and 7S RNA) through the ișe of our automated procedure.

Currently, we are systematically studying mitochondrial rRNA. Our goal is to detect tertiary structure within $16 \mathrm{~S}$ rRNA and quaternary structure between 16S and 23S rRNA. Our ultimate hope is that automated covariation analysis will contribute significantly to a refined picture of ribosome structure.

Our colleagues in biology have begun exr rrit lents to test certain hypotheses suggested by an examination of our program's output. These experiments involve sequencing key portions of the $23 \mathrm{~S}$ ribosomal RNA for species in which the known $16 S$ ribosomal RNA exhibits variation (from the dominant pattern) at the sits of a proposed bonding. The hope is that the $23 \mathrm{~S}$ ribosomal RNA of these species will exhibit corresponding complementary variation or generalized covariation.
\end{abstract}

\section{Introduction}

With the growth and development of sequencing technology, unprecedented volumes of dita have heen made available for analysis. At the same time, new mcthodologies for encoding complex algorithms have been extensively developed and refined. Researchers huve therefore begun to apply the new methodologies to biological computations.

In this paper, we present one such computer application to biology. Specifically, we have been using logic programming techniques in molecular biology to conduct rapid prototyping, to explore altcrnativc hypotheses, and to scan large amounts of genetic data for clues about genetic structure. Working with biologists, we have confirmed the validity of our method with respect to existing biology. More exciting is the fact that our investigations have led to new biology.

\subsection{Initial Focus}

We chose as our initial focus the study of the structure of RNA in the ribosome. The ribosome occupies center stage during protein synthesis. It is the "factory" that synthesizes all proteins. The two main components of the ribosome (the so-called small and large subunits) join together at the beginning of protein synthesis and disengage when synthesis is complete. Yol despite more than thirty years of study, the structure of the ribosome remains largely unknown.

Our automated search for rRNA structure has supplied new hypotheses (potential base-base intcractions) about this structure. Our colleagues in biology are now testing these hypotheses by selectively sequencing additional species. This testing process is described in Section 3. 


\subsection{Choice of Method}

We chose to implement the method of structure search known as covariation analysis [Wocse et al. 1983]. We chose this method for the following three reasons.

First, the biologists who study the structure of the ribosome believe covariation analysis to be the most accurate method of detecting structure in ribosomal RNA. The method of X-ray crystallography is not feasible at present for a structure as large as the ribosome, though it may become so in the near future.

Second, covariation analysis enables us to search not only for secondary structure (i.e., the conventional double stranded helices) but also for tertiary structure (interactions representable only in three dimensions) and quaternary structure (intsractions between molecules) In particular, the method of covariation analysis enables us to scarch for possible interactions betweent the RNA molecules of the two components of the ribosome. Such interactions would be of great interest to those studying the interaction of the two components of the ribosome during protein synthesis.

Third, this method has received little attention from computer scientists, by comparison with the extensive computer studies made of energy minimization, for example [Sankoff and Kruskal 1983, Thanaraj et al. 1989, Le et al. 1989]. Perhaps some have been deterred by the fact that covariance analysis requires extensive use of tree structures and repeated revision of the algorithm to handle increasingly complex biology. Logic programming makes it easier for us to attack such difficulties, as we discuss in Section 7.

\subsection{Results}

Our automation of covariation analysis has already proved successful in several ways. Previously undetected secondary structure has been identified, specifically at positions 63 and 104 of $E$. coli $16 S$ ribosomal RNA. Moreover, new tertiary structure has been discovered in 7S RNA [Pfluger, Overbcek, and Winker 1990]. Finally, indications of possible quatemary structure bonds between $16 \mathrm{~S}$ and $23 \mathrm{~S}$ ribosomal RNA have been detected. If proven by additional experimentation-that is, by selective sequencing of additional species-these would be the first known specific bondings between the two components of the ribosome.

\section{New Structure Detected}

We, tum now to the discussion of a previously unknown covariance in 16S ribosomal RNA that was identified by our covariance detection program. This covariance is between positions 63 and 104 (in $E$. coli) of $16 \mathrm{~S}$ iRNA.

The covariance exhibits the following charac leristics. First, the pairing is Watson-Crick in almost all species investigated. Second, the pairing can $\mid w^{\circ}$ extended to form a helix by adjoining the pair 62 and 105 and the pair 61 and 106. A small amount of covariation evidence exists for each of these additional pairing as well.

We consider first the positive evidence. By far the greatest number of species ( 342 of 375 total) exhibit the pair C.G at positions 63 and 104. These species exhibit the predominant "background of constancy" referred to in [Woese et al. 1983]. Smail, phylogenetically related groups of species exhibit variation from this background of constancy.

The most frequently found pair that varies from the background of constancy is the pair $U, A$. This pair occurs a total of 14 times among the species examined. More compelling is the observation that the 14 occurrences occur in 6 phylogenetically independent groups. Three of these groups are found in the archaebacteria and three in the eubacteria.

One further piece of evidence deserves meption. The pair $A, U$ is also found at positions 63 and 104. This pair occurs twice, in two phylogenetically adjacent cubacterial species, ramely, Streptococcus cremoris and $S$. lactis. Moreover, these two species are phylogenetically adjacent to two of the species that exhibit the pairing $U, A$, namely, $S$. bovis and $S$. salivarius. This double covariation in close relatives is particularly strong support for the proposed interaction. 
One additional circumstance Ifnds support to the proposed bonding. In the phylogenetic subgroup of the genus $M y$ coplasma that consists of $M$. neurolyticum, $M$. hyorhinis, $M$. hyopneumoniae, $M$. sualvi, and $M$. mobile, the pair $U A$ predominates. However, the last species ( $M$. mobile) returns to the predominant pattern $C, G$.

The small amount of negative evidence is confined to individual species in phylogenetic isolation. The pairing $U, G$, acceptable in Watson-Crick pairings in rRNA, $\propto$ curs only three times, each phylogenctically isolated. The pairings $C, A$ and $C, U$ appear in only one isolated $\alpha c$ urrence each. These relatively rare instances of negative evidence are insufficient to offset the overwhelming body of positive evidence.

This new discovery in the wcll-studied 16S rRNA suggests the power of our program to identify additional possible bondings. The program also computes numcrical parameters that suggest the degrees of positive and negative evidence summarized above. We discuss these numerical parameters, both in gencral and as applied to the possible bonding just presented, later in this paper.

\section{Assisting the Biologist}

We now describe in some detail the nature of the assistance provided to the biologist through the use of our program. We consider as a specific context the search for possible covariation between any one of the 1542 positions in 16S rRNA and any one of the 2904 positions in 23S rRNA. (By convention, the position numbering is based on the iRNA sequences of Escherichia coli, whose 16S rRNA and 23S rRNA comprise 1542 and 2904 nucleotides, respectively.) Theoretically, over four million possible pairings exist between these two sets of positions.

From the large number of possible pairings (covarying positions), the program selects several hundred pairs that show some promise. For each, certain parameters of positive evidence and counterevidence are calculated as described below. The output from the program is sorted, based on these parameters, and then is examincd manually. In the case of covariation between $16 \mathrm{~S}$ and $23 \mathrm{~S}$ rRNA, this manual examination reduced the number of pairings to the five to ten most promising pairings. The subsequent investigation focused on these most promising pairings, as we now describe.

For our study, we used alignments of $16 \mathrm{~S}$ and $23 \mathrm{~S}$ rRNA. Woese made available his alignment of the 16S rRNA sequences, comprising nearly 400 eubacterial and archaebacterial species. We were also able to use R. R. Gutell's alignment of the 23S rRNA sequences for some 30 archaebacterial and eubacterial species, as well as certain $16 \mathrm{~S}$ sequences aligned with $E$. coli. Note the key point that many more sequences are available for 16S rRNA than for $23 S$ rRNA.

We instructed our program to search for evidence of covariation between positicns in the $16 \mathrm{~S}$ alignment and positions in the $23 \mathrm{~S}$ alignment described above, which limits the search tos the relatively small subset of species for which both 16S and 23S rRNA sequences are known. (The program can also be instructed to search for covariation within 16S rRNA alone, within 23S rRNA alone, within the archaebacteria alone, or within the eubacteria alone.)

As a result of its search, the program specifies a set of pairs of numbers, each pair indicating a possible instance of covariation. One initially promising possible covariation turned up by our program involved position 943 of $16 \mathrm{~S}$ rRNA and position 2330 of 23S rRNA.

This possible covariation opened the way for our biological colleagues to modertake new sequencing efforts which we now discuss. As stated above, the number of known $16 S$ rRNA sequences greatly exceeds the number of known 23S rRNA sequences. This additional knowledge of the 16S rRNA sequences makes it possible to select organisms whose 16S rRNA (already known) and 23S rRNA (when sequenced) would provide evidence that would test (confirm or disprove) the suggested covariance.

The following steps have the objective of selecting certain crucial species for additional sequencing. First, one position of interest in 16S rRNA-for example, position 943-is considered. Second, the predominant base at that position of interest is determined- $U$ in our example. Third, those species are considered for which 16S rRNA data are available but 23S rRNA data are not. Those species are divided into two sets, namely, the set of species that exhibit the predominant base ( $U$ in our example) at the position of interest, and the set of species that exhibit other than the predominant base at that position. The species that exhibit the predominant base are said to exhibit constancy; the remaining species are said to exhibit 
variation. Fourth, pairs of close rclatives are sought in which onc species of the pair exhibits constancy and the other exhibits variation at the position of interest. These pairs of ciose relatives are the crucial species to sequence. Using pairs of closcly related sequences reduces the possibility of fortuitous covariance matches.

The crucial pairs of species thus chosen are then examined as follows. Species that show variation in the 16S rRNA (at the position of interest) are sequenced, in order to test whether a corresponding variation $\propto$ ccurs in the 23S rRNA (again at the position of interest, in this case position 2330). Close relatives that show constancy in the $16 \mathrm{~S}$ are also sequenced, in order to test whether they also show constanc! in the $23 \mathrm{~S}$. In this way the investigator determines, to a high degree of likclihood, whether the specics that show variation in the $23 \mathrm{~S}$ are those species and only those species that show variation in the $16 \mathrm{~S}$. The investigator chooses for examination only those $23 S$ rRNA examples that would provide a critical test of (provide additional evidence favoring or, alternatively disproving) the suggested covariance.

The sequencing of the $23 \mathrm{~S}$ rRNAs is simplified by the fact that it is not necessary to sequence the entire molecule to test a possible covariance. Since the molecule is sequenced directly (rather than sequencing its :loned gene), it is necessary to sequence only that region of the molecule that contains the position in question. This task is accomplished by selecting from an existing set (or, if necessary, synthesizing) the proper "primer" that initiates the sequencing reaction (a template copying process) at a point near the position in question (within a few to a few hundred nucleotides). Thus each test is accomplished by sequencing less than $10 \%$ of the $23 S$ rRNA molecule. For the case in point, an existing primer that initiates (backward) synthesis at position 2438 in $23 \mathrm{~S}$ rRNA was used, which easily covers the position of interest, 2330.

By contrast, the sequencing of an entire $23 S$ molecule would require more than ten times the effort. 'Thus, our computational techniques enable biologists to pinpoint their efforts on certain crucial species and on a key portion of the molecule, saving literally years of (otherwise unguided) work.

We regret to state that the specific possible bonding discussed here was not supported by the additional experimental evidence thus gathered. However, it is only from such a set of possible quaternary interactions that the true interactions can be ultimately extracted.

\section{Covariation}

In this section we present the notion of covariation [Woese et al. 1983], also known as covariance, which is implemented in our program. To discuss this notion, we first consider an alignment of corresponding sequences for a number of organisms, related closely or distantly as the case may be.

In the study described here, we use an alignment of scquences for the small subunit of ribosomal RN:A (16S rRNA in bacteria) in species ranging throughout the bacterial kingdoms of archaebacteria and eubacteria. This wide range of distantly related species is appropriate to the sequence material employed. Ribosomal RNA appears in all bacterial and eukaryotic specics and is believed to serve similar functions in all species (although the precise functioning of this RNA is not yet known). Therefore, it is perhaps not surprising to find that at least some portions of the small subunit rRNA molecule are highly conserved not only from species to species but also from kingdom to kingdom. By contrash a covariation-based study of material specific to one phylogenetic subgroup would range at most over species in that subgroup.

The use of an alignment as a basis for defining covariation leads to the use of the following terms. The term column (or position) refers to one column of an alignment of a set of corresponding sequences for various species. The $k$ th column consists of the values of the $k$ th position in the alignment for each of the organisms in the alignment. In one of the alignments employed, there are 375 sequences, and the columns are numbered from 0 to 1847. In this case, each column contains 375 entries, one for each bacterial species considered. We conventionally reserve the first entry in each column for $E$. coli.

Each entry in a column is one of the following characters: $A$ (adenine), $G$ (guanine), $U$ (uracil), $C$ (cytosine), an indel (a dash, which indicates that there is no entry in this column for this organism), $N$ (a base is present in at this position, but its identity is unknown), or any of the standard codes for ambiguous bases. The characters of indel, $N$, and ambiguity code are all trcated identically by our algorithm. 


\subsection{Watson-Crick Covariation}

We now discuss the notion of Watson-Crick covariation, before tuming to the notion of generalized covariation. We present Watson-Crick covariation in terms of the notion of complementary columns. Two columns $x, y$ of an alignment (of RNA sequences) are complementary if the corresponding entries for each specics are complementary. An entry of $A$ complements $U$, while $C$ complements $G$. (Complementarity is symmetric, so that $U$ complements $A$ and $G$ complements $C$ as well.) Also, $G$ complements $U$ weakly, and this combination is allowed in complementary columns. Finally, an indel complements an indel, and any ambiguity code complements any base or any other ambiguity code, for the purposes of our algorithm. (Certain pairs of ambiguity codes cannot in fact be complementary, but ambiguity codes cher than $N$ are rare in the data we employ. The corresponding modification of the algorithm would bring little improvement in its performance, in our vicw.)

We are now ready to introduce the notion of covarying columns (or covarying positions). An individual column of the alignment may contain the same base for all organisms in the alignment. For example, all entries in the colurnn may be $A$. Such a column is termed invariant.

On the other hand, an individual column of the alignment may vary from organism to organism. For example, the column may contain an cntry of $A$ for some organisms and an entry of $U$ for others.

It is relatively easy for two nonvarying columns to complement one another. For example, any column of all $A$ complements any column of all $U$. However, this complementarity dees not necessarily associate a particular column of all $A$ with a particular column of all $U$.

However, if two columns of the alignment vary so as to complement each other for all organisms in the alignment, there is significant cvidence for the pairing of the two cctresponding positions in the actual physical structure of the RNA molecule. Consider, for example, the following pair of columns.
A U
A U
C $\mathbf{G}$
C $\mathbf{G}$
U A

These two columns are called covarying because the variation in one column is echoed complementarily in the other column.

\subsection{Generalized Covariation}

Generalized covariation is said to occur when two columns vary in corresponding ways, but with a pairing other than the usual Watson-Crick pairing. For example, the following two columns exhibit generalized covariation.
$\mathbf{U} \mathbf{U}$
U U
C C
C C
U U

In this case the pairing rules are that $U$ pairs with $U$ and $C$ pairs with $C$. This set of pairing rules is encountered, for example, in positions 1307 and 1330 of $E$. coli 16S ribosomal RNA, and in the corresponding positions in other species. Such nonstandard pairing rules are thought to correspond to unusual struct.rris's or positioning in the three-dimensional molecule, such that the nonstandard pairing is more functional than standard Watson-Crick pairing would be at that particular location. For example, a nonstandard pairing might impart an extra bend or twist to a portion of an RNA molecule.

\subsection{Evolutionary Interpretation of Covariation}

The following evolutionary interpretation of covarying columns can be offered. Consider any two covarying columns, which we shall refer to as the first and second columns, respectively. In an original species, the first position (column) may have contained $A$ and the second $U$. (Actually we may have no 
way of knowing the original values.) These two positions bonded to one another in the corresponding RNA of that species.

Then, $A$ mutated to $C$ in the first position. As a result, the two positions were no longer complementary and could no longer bond. This mutation altered the sccondary structure of the RNA, and the ritosomes containing it were less effective. Fortunately, most organisms have multiple copies of the coding for ribosomal RNA. Thus only some ribosomes, those made from the mutated copy, were less effective. Later, $U$ mutated to $G$ in the second position. This mutation restored complementarity of the two positions, restored their bonding, and made the ribosomes more effective once again. What we now see are only the most effective versions of the ribosomal RNA, those in which the two positions in question are complementary.

\section{An Automated Procedure for Covariation Analysis}

In this section we present an automated method for finding possible base-base interactions in ribosomal RNA. Our method is based on the notion of covariation [Woese et al. 1983], which was presented in the preceding section. We begin with a brief overview of our method.

Our automated procedure for covariation analysis begins by assessing, at each position in the alignment, the presence or absence of change at that position in various species. Change is assessed with respect to the predominant base at each position. Change is also assessed within the context of the phylogenetic tree for those species whose sequences are available. (The use of the phylogenetic tree contributes to the accuracy of the picture of change thus developed, as explained below.) The result of this assessment is a set of "change lists", each listing the phylogenetic points of change for one position in the alignment of sequences.

The change lists thus obtained are then compared for each pair of positions in the alignment. Any two positions that have similar change lists are likely to exhibit covariation. These promising pairs are passed to the next step.

The program examines each of the promising pairs in greater detail to assess two kinds of evidence: positive evidence that supports the hypothesis of covariation between the two positions, and negative evidence against covariation between the two positions.

The biologist then manually examines the most promising covarying pairs. In scme cases, the evidence presented by the program is sufficient to establish covariance (and, by implication, bonding) at the positions indicated. The evidence is sufficient, for example, in the case of the pair 63,104 discussed earlier. In other cases, more evidence is required. In such cases, the biologist may gather additional sequence data to establish or refute the possible covariation. The sequencing effort is focused on the most promising positions pointed out by the program, and on those species most likely to support or refute covariation at the positions in question.

As mentioned above, covariation is most accurately assessed in the light of a phylogenetic tree for those species for which sequence data are available. The purpose in using the phylogenetic tree is to assess how many phylogenetically independent events of covariation have occurred. For example, if all archaebacteria differ from all eubacteria at a given position in an RNA sequence, this represents essentially one event of variation, even though the effects appear in many species. The phylogenetic tree is used in the first step of our procedure, to which we now turn.

\subsection{Change List at One Position}

We begin by determining the change list at one position in an alignment. The change list depends on hoth the base at the given position in each species and the phylogenetic tree of all of the species under consideration. We wish to determine which phylogenetic subgroups (subtrees of the phylogenetic tree) exhibit change at the given position, and which do not. As an example, consider the following phylogenetic tree of archaebacteria. 


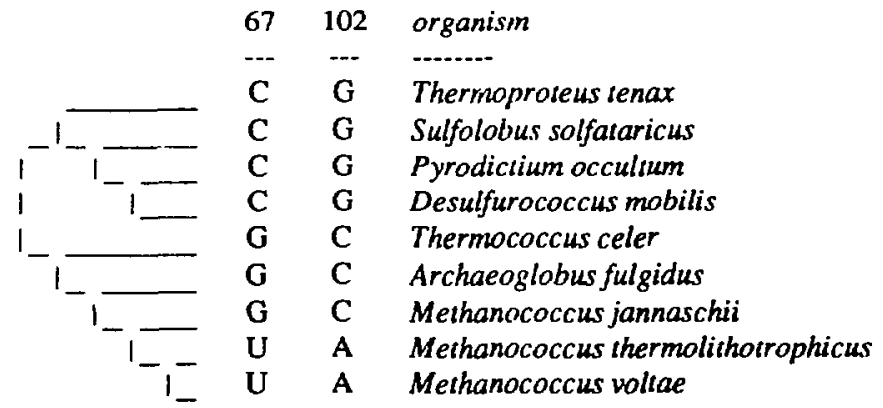

This tree supplies the branching pattern, the contents of positions 67 and 102 of an alignment, and the names of the organisms. (The numbers 67 and 102 refer to the corresponding positions in the sequence for $E$. coli. Note that at present the program assumes a binary tree.)

To compare the patterns of change at two different positions (such as 67 and 102 in the example), we need a succinct way of desc." g a pattem of change. Consider first the pattern of change at position 67.

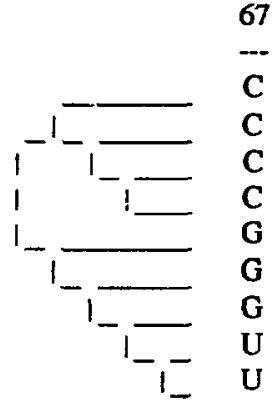

Our progranı examines each internal node of the phylogenetic tree in turn. (In the example, the internal nodes are denoted by vertical lines.) At preseni, the program assumes that each internal node has two subtrees. For example, the leftmost or "root" node in the tree above has two subtrees of four and five organisms, respectively.

At each internal node, the program determines which base $(A, C, G$, or $U)$ predominates within the node's two subtrees. Predominance is determined by counting the occurrences of each base. In the example, the base $C$ predominates in the first subtree of four organisms, while the base $G$ predominates in the second subtree.

If the predominant base changes from cne subtree to the other, we say that a change occurs at the node that joins them. In the example, a change occurs at the root node. If the predominant base is the same in both subtrees, we say that no change occurs at the joining node. Of course, the definition of change is made with respect $w$ a chosen position in the alignment. The same node in the phylogenetic tree may exhibit change at one position in the alignment but not at another.

In order to record which nodes exhibit change, it is useful to number them. Our program numbers the nodes sequentially, assigning the lowest numbers to the top subtree and the highest numbers to the bottom subtree, as illustrated below. However, any numbering scherie would suffice. 


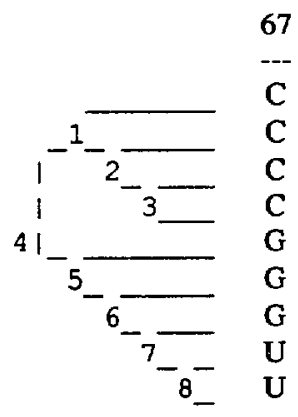

At position 67 in the example, changes occur at nodes 4,6 , and 7 . In arriving at this list of changes, it is necessary to consider a subtree in which two bases occur with equal frequency. The second subtree of node 5 contains two occurrences of $G$ and two occurrences of $U$. In this case, both are considered to predominate, and if either is predominant in the other subtree, no change is said to occur. In the example, the base $G$ also predominates in the other subtree of node 5 , and so no change is said to occur at node 5 .

Indels and indeterminate bases are counted as zero; they do not contribute to the count for any base. In a subtree with no specific base data, all bases occur zero times, all are considered equally predominant, and no change is recorded between that subtree and another subtree, whether or not the other subtree has specific base data.

\subsection{Comparison of Change Lists}

Once a change list has been determined for each position in the alignment, the program is ready to examine pairs of positions for possible covariation. For each pair of positions, the corresponding change lists are compared. The parameters of interest are the following: (1) the number of entries (changes) in the larger list; (2) the number of matching entries in the two lists (the number of nodes in the phylogenetic tree at which both positions in the alignment exhibit change-ihat is to say, possible instances of covariant change); and (3) the percentage of agreement, that is, the number of matching entries expressed as a percenlage of the number of changes in the larger list. In the example, the change lists for positions 67 and 102 each contain three entries, and there is $100 \%$ agreement between the two lists.

Pairs of alignment positions showing less than 50\% agreement are discarded. (The 50\% threshold can be adjustea by the user.) Of over a million possible pairs in 165 ribosomal RNA, fewer than 800 pairs were retained by use of this test. The pairs thus retained are then sorted by descending percentage of agreement.

The highest percentages of agreement in $16 \mathrm{~S}$ ribosomal RNA correspond to known secondary bonds and to known examples of tertiary bonding and/or generalized (i.e., non-Watson-Crick) covariation. A total of 281 known secondary bonds is found among the pairs retained.

A number of pairs of somewhat lesser agreement were taken as candidates for new hypothetical bondings. For each of these pairs, the phylogenetic tree was printed out, with the corresponding base at each of the two positions displayed alongside each species. These trees of pairs were then examined manually to judge the degree of covariation.

\subsection{Numerical Assessment of Evidence}

Although strong covariation between two positions generally leads to similar change lists at those two positions, the reverse is not necessarily true. That is, two positions may have similar change lists but still fail to covary. For example, two invariant positions will have identical change lists of length zero. As another example, consider those positions that differ from the archaebacteria to the eubacteria but are invariant within each kingdom. Each of these positions will have an identical change list of length one. Many more examples involving longer change lists are encountered in practice.

For these reasons, those pairs of positions that successfully pass the large volume screening for similar change lists are then examined individually, pair by pair, to assess the evidence for and against covariation in a more refined (and compulationally intensive) manner. 
Each pair of positions that have similar change lists is processed as follows. First, the program obtains, for each species in the phylogenetic tree, the base prescnt at each of the two positions in the alignment.

\begin{tabular}{|c|c|c|c|}
\hline & 67 & 102 & organism \\
\hline & -- & --- & ------- \\
\hline & C & G & T.tenax \\
\hline & C & G & S. solfataricus \\
\hline & C & $\mathbf{G}$ & P. occultum \\
\hline & $\mathrm{C}$ & $\mathbf{G}$ & D. mobilis \\
\hline & G & C & T. celer \\
\hline & G & C & A. fulgidus \\
\hline & $\mathbf{G}$ & C & M. jannaschii \\
\hline & $\mathrm{U}$ & A & M. thermolithotrophicus \\
\hline & $\mathrm{U}$ & A & M. voltae \\
\hline
\end{tabular}

In the example repeated here, the bases at positions 67 and 102 are retrieved for adch species. From the species $T$. tenax, for example, the bases $C$ and $G$ are retrieved to form the pair $C, G$. These pairs of bases are then mapper onto the phylogenetic tree, to yield a tree of pairs. (The actual species rames are omiucd at this point.)

Next, the tree of pairs is "collapsed" to eliminate repeated adjacent occurrences of the same pair. In the example above, the four adjacent $\alpha c$ urrences of the pair $C, G$ are merged as follows.

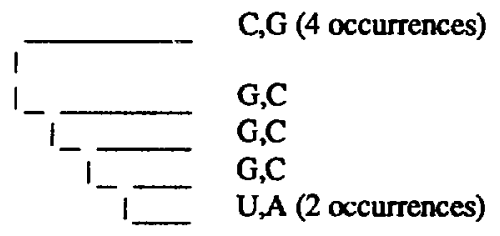

The two adjacent occurrences of $U, A$ are also morged. At each such merged node, a count is maintained of the number of pairs merged there.

The program also performs the following additional type of merging. Whenever a subtree is encountered that consists of repetitions of the same predominant pair, except for a different pair at the deepest level, a "predominance structure" is built. The predominance structure indicates the predominant pair first and the exceptional pair second, as follows.

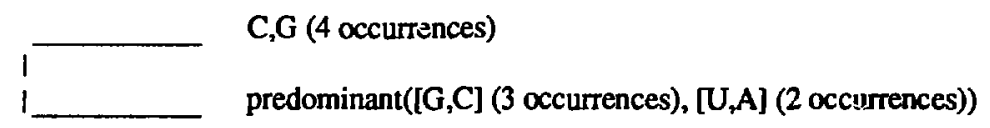

In addition, in large trees two adjacent predominance structures that have the same predominant pair are further merged. The combined predominance structure includes the predominant pair, the count of its occurrences, and a list of the exceptional pairs and their counts.

In many cascs, the collapsed tree is small, simple, and easily read. But not all trees collapse into such a convenient form. How are we to process the trees that renain large? We have found, in practice, that a strongly covarying pair generally yields a small collapsed tree. By contrast, a tree that remains large even after collapse generally represents noncovariant variation in two positions of interest.

The program then calculates a set of numerical parameters from the collapsed tree. These numerical parameters suggest the degree of evidence for covariation and the degree of counterevidence. (The definitions of these parameters are by no means final. In fact, we welcome suggestions as to additional parameters and refinements of the parameters defined below.)

The degree of positive evidence is suggested by the following two parameters. First, the number of phylogenetically distinct occurrences of a given pair suggests how much variation exists. Covariation can be detected only when variation is present. Multiple phylogenetically distinct occurrences of a given pair 
suggest a potential, at leasi, for multiple pieces of positive evidence. We count phylogeneticaily distinct occurrences simply by counting the number of times a given pair is listed within the collapsed tree. The program prints out the maximum over all 16 possible base pairs of the number of phylogenetically distinct occurrences. In the example, this maximum is one, because no pair occurs more than once independently. Larger values for this maximum are oblained when more species with additional variation are present.

The program also counts, as a second parameter of posilive evidence, how many of the 16 possible base pairs exhibit at least two phylogenetically distinct occurrences in the tree. In the example, this count is zero, because no pair occurs more than once independently. Larger values for this count are obtained when more species with additional variation are present.

The degree of negative evidence is suggested by two parameters that we now describe. By way of introducing these parameters, we suggest that noncovariant change (that is, change in one position but not in the other) tends to indicate a lack of covariation. We then define parameters based on such noncovariant change.

The program begins the assessment of negative evidence by filling in a four-by-four matrix to indicate which pairs are present in the tree and which are not. The four-by-four matrix for the example given above is as follows.

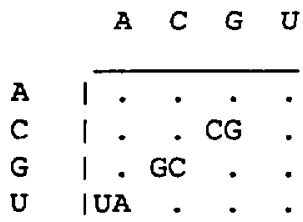

The first parameter for negative et idence is the notion of "collision." A collision is present in a row or column if that row or column has more than one entry. In the above example there are no collisions, because each row and each column contain at most one entry. However, in the following example several collisions occur.

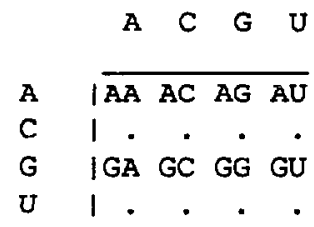

We could describe this example as follows. The first position is either $A$ or $G$, and the second position can be any base at all. There is no covariation, and the count of collisions supports tiis. We count one collision in each of the four columns, for a total of four. In the first row we count three collisions: the number of entries (four) minus one. In the third row we also count three collisions. The total number of collisions is ten.

Our second parameter for negative evidence is simply a refined version of the notion of a collision. Instead of counting all collisions, we count only what we call "solid collisions," which we define as follows. We begin with the notion of "solid su.port" for a pair of bases. A pair of bases at a pair of positions in the alignment has solid support if that pair occurs at least twice in phylogenetically adjacent species. In terms of the collapsed tree discussed above, a pair of bases has solid support if the count of merged species associated with some occurrence of that pair is two or more. In the example of positions 67 and 102 given earlier, all three pairs $C . G ; G, C$; and $U, A$ have solid support. We then fill in the four-by-four matrix with pairs that have solid support, and count the number of collisions associated with the resulting matrix.

The intuition behind the notions of solid support and solid collision is as follows. An isolated occurrence of an uriexpected base pair may not constitute solid evidence against covariation. Consider, for 
example, the appearance of an unexpected pair $A, A$ in two positions that otherwise follow the Watson-Crick pairing rules. This unexpected pair might appear for any of the following reasons. First, a relativciy unstable configuration might be present in one isolated species, without being carricd along to other species. Second, an error in sequencing might occur (for example, when chemical bonding effects, such as those sometimes present near hairpin loops, affect migration rates and niake the sequencing gel more difficult to read). Third, an error might arise in the course of aligning sequences (as when aligning sections that are highly variable, including length variations). For these reasons, when assessing negative evidence we seek a procedure that is relatively insensitive to isolated errors of the types just mentioned. The count of solid collisions is intended to serve as such a procedure.

In fact, we have found pairs that show substantial positive evidence of covariation and no solid collisions to be quite promising upon manual examination, even when collisions of an isolated, nonsolid nature are present.

\subsection{Example of Numerical Assessment of Evidence}

We turn now to the numerical parameters for positive and negative evidence calculated by our program for the pair of positions 63,104 discussed earlier in this paper.

The parameters for positive evidence are as follows. The maximum number of phylogenetically distinct occurrences of a pairing is the six occurrences of $U, A$. This number suggests the potential for the considerable positive evidence that we find on manual examination. The number of pairings that occur more than once phylogenetically is three: $C, G ; U, A$; and the isolated occurrences of $U, G$.

The numerical parameters for negative evidence at positions 63 and 104 are as follows. The number of solid collisions is zero, indicating no solid counterevidence. The corresponding four-by-four matrix is the following.

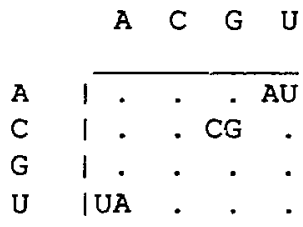

The total number of collisions, solid and otherwise, is six, as indicated by the following four-by-four matrix.

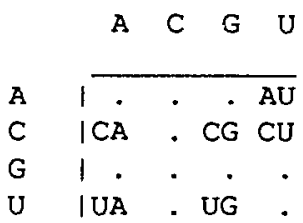

The substantial count of six collisions, in a covariance for which there is strong evidence, suggests the relative inapplicability of the count of total collisions, by contrast with the more relevant count of zcro solid collisions.

This concludes the discussion of our algorithm. We proceed new to discuss the validation of this algorithm with respect to known biology, the known aspects of structure in ribosomal RNA.

\section{Validation}

Before we used the automated covariance procedure described above to seek new (previously unknown) structure, we validated the procedure with respect to known bondings. Specifically, we used the secondary structure proposed in [Woese tt al. 1983], and most recently revised in [Gutell and Woese 1990). as the basis for validation. 
We present the validation of the automated procedure in four subsections. In the first subsection, we stress the need for accurate detection, especially when considering tertiary and quatemary structure. In the second subsection, we present the validation of a covariance-based method for detection of secondary structure, and compare the performance of this method with that of other published methods. In the third subsection, we present the validation of automated covariance analysis in the context of a phylogenetic tree. Finally, in the fourth subsection, we present the validation of automated covariance analysis in the detection of tertiary structure and generalized covariation. Note that validation of search methods for quaternary structure (bonding between the $16 \mathrm{~S}$ and $23 \mathrm{~S}$ RNA molecules) must be based on the corresponding validation for tertiary structure and generalized covariance, because no quaternary bondings are known as yet.

\subsection{The Need for Accurate Detection}

The list of known tertiary structure in 16S rRNA is short, by contrast with the hundreds of bonds in the known secondary structure. The relatively few tertiary bonds must be sifted from over one million theoretically possible pairings within the set of 1542 positions in $E$. coli. In particular, we note that positions already bonded in secondary structure must still be included in the search for tertiary structure. The reason for such inclusion is that one base can be bonded to two other bases. Such triples of bases are known in the three-dimensional structure of transfer RNA detcrmined by X-ray crystallography [Watson et al. 1987, p. 389].

Accuracy of detection in the search for tertiary structure is crucial because the few bonds of interes! must be detected out of a great many possible pairs. Even a $1 \%$ rate of "false positives" would turn up ten thousand spurious pairings out of the million theoretically possible-far too many to sift through by hand to find the few true pairings. Accuracy of detection is even more crucial in the detection of quaternary structure, that is, bondings between the $16 \mathrm{~S}$ and $23 \mathrm{~S}$ rRNA. More than four million pairings are theoretically possible, but at most a few of these, in all likelihood, actually bond.

\subsection{Validation for Detection of Secondary Structure}

Our first validation test used automated covariance analysis to detect known secondary structure in $16 \mathrm{~S}$ eubacterial rRNA. We applied this procedure to the following three phylogenetic groups. The first group consisted of 16 eubacteria that ranged from close relatives of $E$. coli to distant relatives. The second group consisted of $E$. coli and its four closest relatives from the first group. The third group consisted of 5 phylogenetically widely distributed eubacteria from the first group. Within each of these groups, our procedure extended helices to include adjacent complementary positions, even when those positions were invariant and thus lacking in supporting covariance data. This approach led to an increased percentage of prediction at the expense of some error in overextending helices. We made relatively litule use of phylogenetic data at this stage.

By combining the bonds detected in the three groups mentioned above, the procedure detected a total of $65 \%$ of the bonds in the known secondary structure. This detection rate is comparable with the best algorithms for secondary structure described to date [Thanaraj et al. 1989, Le et al. 1989].

Of even greater imponance for subsequent steps was the low error rate obtained: only $5 \%$ of the predicted bonds were in error. Furthermore, these errors involved only the erroneous extension of otherwise valid helices. No entirely spurious helices were "detected." This high level of accuracy promised well for the more demanding searches for new secondary structure, generalized covariance, tertiary structure, and eventually quaternary structure. (By contrast, the best automated predictions we are aware of [Thanaraj et al. 1989] have an error rate of over 25\%).

We auribute our high detection rate, coupled with the low error rate, to the use of covariance analysis to detect secondary structure. The biologists who study ribosomal structure have found covariance analysis to be the most accurate structure detcction method available. (The method of $\mathrm{X}$-ray crystallography, which is still inore accurate, is not feasible at present for a structure as large as the ribosome.)

We remark that other methods [Sankoff and Kruskal 1983], and in particular those of Thanaraj et al. [1989], use a great deal of additional (non-sequence) evidence-for example, results from chemical and enzymatic probes of ribosomal RNA. Such information requires many time-consuming experiments to obtain, and the results are often difficult to interpret. The covariance procedure presented here, on the other hand, requires only the sequence data for the species in question and for a small number of related species. 
Another recent study [Le et al. 1989] does not present specific data regarding 165 ribosomal RNA, and so we cannot make a specific comparison of accuracy. It is interesting to note that their method involves a seeking of "consensus" among the sequences and structures for several species. This consensus may recover some but not all of the information that is available through covariance analysis.

In concluding the discussion of validation for secondary structure, we wish once again to stress the accuracy of predictions made by our procedure. Accuracy is even more critical in tertiary and quaternary structure detection than in detection of secondary structure.

\subsection{Validation for Covariation in a Phylogenetic Tree}

Our next validation test involved the use of many more species- -375 cubacteria and archacbacteria in all-in conjunction with a phylogenetic tree for those species. (The procedure for use of a phylogenetic tree in covariance analysis is discussed in Section 5 above.) Our procedure constructed a "change list" for each position in 16S rRNA, compared the change lists for each pair of positions, and soried the resulting pairs by descending percentage of agreement and descending length of the longer change list. Positions that exhibited fewer than two changes were omitted, as were pairs showing less than $50 \%$ agreement.

The greatest percentages of agrecment corresponded to known bonds. A total of 281 known bonds of secondary structure was detected, with change lists agreeing $50 \%$ or more. We then filtered the known bonds out of the program's outpul, and examined what remained. High percentages of agreement were indicated for two examples of alternative bondings, namely, positions 367 and 393 (an alternative to 368 with 393) and positions 596 with 644 (an alternative to 595 with 644). Our biological colleagues confirmed the viability of these alternatives in the light of current knowledge.

\subsection{Validation for Tertiary Structure and Generalized Covariance}

In our third validation test, alitomated covariance analysis was used to detect known tertiary structure and generalized covariance. These categories involve the following increased difficulties. First, pure Watson-Crick bonding cannot be assumed. For example, there are pairs of positions that pair as $A, G$ or $G, A ; U, U$ or $C, C$; and even $A, A$ or $G, G$. (These unusual pairings are exclusive of Watson-Crick bonding. For exampie, the options $U, U$ or $C, C$ represent the only pairs that occur at a given pair of positions. It is thought that these unusual pairings improve the functioning of the rRNA molecule by imparting an extra bend or twist at a given location.) The second increased difficulty is that the secondary structure imposes little constraint on the possible tertiary structure. Thus a graph theoretic or "divide and conquer" approach [Thanaraj et al. 1989, Le et al. 1989] cannot be applied to tertiary structure.

The automated covariance procedure detected $4:$ following known features of teriary structure and generalized covariance. Positions 245 and 283 exhibıt either $U, U$ or $C, C$ pairing, but not Watson-Crick pairing. Positions 570 and 866 bond in tertiary structure in a Watson-Crick manner. Positions 722 and 733 exhibit either $A, A$ or $G, G$ pairing, but not Watson-Crivk pairing. Positions 779 and 803 pair in a WatsonCrick manner. Positions 1307 and 1330 , like positions 245 and 283 , exhibil only $U, U$ or $C, C$ pairing. Positions 1357 and 1365 exhibit $A, G$ or or $G, A$ pairing in the eubacteria, but pair in a Watson-Crick manner in the archaebacteria. All of these features were detected by our automated procedure.

The automated covariance procedure also detected the covariation present in two "tetra loops," those at positions 83 through 86 and 1029 through 1032. These hairpin loops consist of only four base positions. The presence of such a tight loop is thought to constrain the possible sets of bases that can appear within the loop. Our procedure detected evidence for the presence of such constraints.

Finally, a variation on our procedure, modified to detect covariation between contiguons triples of positions rather than between single positions, detected the known tertiary helix between positions 505-507 and 524-526.

The three validation tests presented here set the stage for the discoveries of new (previously unknown) structure described in the preceding sections. 


\section{Programming Methodology}

The investigation described in this paper benefited greatly from a number of features and facilities provided by logic programming. Among the most valuable is the capability for rapid prototyping in logic programming. Rapid prototyping refers to the ease with which a desired algorithm and variations on it can be quickly programmed (if not in the most efficient form) and tested immediately, and to the ease with which modifications are made. Ease of modification proved most important in our work, because we found it necessary to go through a series of modifications in order to arrive at the current forms of our programs. These modifications were not merely corrections. Each stage of modification brought additional insight into the problem and provided valuable experience. As a result, the final forms of the programs are remarkably different from the initial versions. We now give examples of the kind of modifications that we needed to make $\rightarrow$ modifications that were made easier by the capability for rapid prototyping.

Our first example of a potentially major modification concerns a program originally developed to process single occurrences of bases in a sequence. This program was adapted in a single day and run in a modified version that processes triples of bases in an analogous manner. Output from the modified version alerted us to a deficiency in the original version, which was then also rethought and reworked.

A second example of the need for substantial reworting of our programs, reworking that was facilitated by rapid prototyping, involves the handling of conficting indications and evidence. For example, if evidence is present for the bonding of both position 63 with position 104 and position 65 with 104, a decision may be nceded as to which of these possible bondings is betuer supported.

Early versions of our programs attempted to choose between such alternatives in an automated fashion. More recently, we have recognized that many such judgments must be made by the biologist. The biologist often has additional sources of information that arc unavailable to our program. For example, certain base positions in a sequence may appear transposed because of sequencing phenomena such as "band compression." This phenomenon is encountered in certain subsequences of certain species when multiple occurrences of $G$, guanine, are present. This pntential source of error is known to the biolegist and is to be addressed by alternative but more difficult sequencing techniques only if that subsequence of that spccies becomes a focus of interest. A computer run can identify that subsequence as counterevidence to a bonding. However, the program is not equipped to perform the necessary resequencing to resolve the issuc. Instead, we use rapid prototyping to implement and test various measures of positive and negative evidence to identify the items most needing the biologist's attention.

A third example of the need for rapid prototyping, occasioned by the need for substantial and repeated modification, is the following. We recognized early in our investigation that the assessment of covariational evidence in the context of a phylogenetic tree (for the organisms involved) would be crucial to our investigation. It was not clear what the exact algorithm for such assessment would be. Consequently, we encoded many versions of assessment procedures in our various programs. These versions were easily implemented through the use of logic programming, and they were crucial to our successful experimentation.

The ease with which we were able to introduce new algorithms and modify existing code was due, largely, to the facilities for manipulating lists and trees. Since many aspects of our algorithm required careful maintenance and examination of trees, these facilities provided substantial convenience.

It is, perhaps, worth noting that the programs that we created were developed on Sun workstations but were written in a dialect of logic programming that allows use of multiprocessors. That is, for the most lime-consuming types of computations, we now have the capability of moving the code directly to parallel processors (in [Foster, Overbeek and Winker 1990] we cover this aspect of the technology that we are developing.)

\section{Conclusions}

Covariation techniques [Woese et al. 1983] have served as a reliable predictor of secondary structure for a number of years. Automation of the computational search for covariation has enabled us to examine much more data than could be practically examined manually. We now have the ability to scan large volumes of data. This ability has led to conjectures concerning quaternary structure (the connections 
between molecules such as ribosomal 16S and 23S RNA). These conjectures have enough evidence to support them that biologists are now formulating and carrying out experiments to tests these conjectures.

We have also used our program to derect new secondary structure. Specifically, we hypothesize, based on output from our program, hydrogen bonding betwecn positions 63 and 104 of E. coli $16 S$ ribosomal RNA (and between corresponding positions in nther bacterial species). We also hypothesize the extension of this pair to a helix including positions $6 i-63$ paired with 104-106. " method similar to the one presented here has been used to detect a new possible tertiary bonding in 7S bucterial RNA [Pflugcr, Overbeek, and Winker 1990].

Our programming methodology specifically employs logic programming in Prolog and Strand [Foster and Taylor 1989]. This methodology has brought two major benefits. First, the capability for rapid prototyping embodied in logic programming has enabled us to refine our algorithm through repeated generations. Second, in a limited number of cases, the ability to exploit parallel computation has also been useful [Foster, Overbeek, and Winker 1990].

\section{Acknowledgments}

We acknowledge those who provided the data without which this work would not have been possible. The sequence and aligriment data for $16 S$ rRNA were supplied by C. R. Woese. We also thank R. R. Gutell, who supplied the sequence and alignment data for $23 \mathrm{~S}$ rRNA and certain $16 \mathrm{~S}$ data as well. The programming tools used for sequence manipulation and analysis were developed by $R$. Overbeek and others [Foster and Taylor 1989].

\section{References}

[Foster, Overbeek, and Winker 1990] Foster, I., Cverbeek, R., and Winker, S., Argonne National Laboratory, unpublished information.

[Foster and Taylor 1989] Foster, I., and Taylor, S., Strand: New Concepts in Parallel Programming. Prentice Hall, Englewood Cliffs, New Jersey.

[Gutell and Fox 1988] Gutell, R. R., and Fox, G. E., A compilation of large subunit RNA sequences presented in a structural fonnat. Nucleic Acids Research 16, supplement, R175-R269.

[Guttll et al. 1986] Gutell, R. R., Noller, H. F., and Woese, C. R., Higher order structure in ribosomal RNA. EMBO Journal 5, ‘111-1113.

[Gutell et al. 1985] Gutell, R. R., Weiser, B., Woese, C. R., and Noller, H. F., Comparative anatomy of 16S-like ribosomal RNA. Prog. Nucleic Acid Res. Mol. Biol. 32, 155-216.

[Gutell and Woese 1990] GutelI, R. R., and Woese, C. R., Higher order structural elements in ribosomal RNAs: Pseudo-knots and the use of non-canonical pairs. Proc. Natl. Acad. Sci. (USA), in press.

[Haselman et al. 1989] Haselman, T., Gutell, R. R., Jurka, J., and Fox, G. E., Additional Watson-Crick interactions suggest a structural core in large subunit ribosomal RNA. J. Biomol. Structure and Dynamics 7, $181-186$.

[Le et al. 1989] Le, S.-Y., Owens, J., Nussinov, R., Chen, J.-H., Shapiro, B., and Maizel, J. V., RNA secondary structures: Comparison and determination of frequently recurring structures by consensus. Comp. Appl. Biosci. 5, 205-210.

[Noller and Woese 1981] Noller, H. F., and Woese, C. R., Secondary structure of 16S ribosomal RNA. Science 212, 403-111. 
[Noller 1984] Noller, H. F., Structure of ribosomal RNA. Ann. Rev. Biochem. 53, 11'?-162.

[Nussinov and Tinoco 1982] Nussinov, R., and Tinoco, I., Jr., Secondary structure model for the complete simian virus-40 late precursor RNA. Nucleic Acids Research 10 (1), 351-363.

[Olsen 1983] Olsen, G. J., Comparative Analysis of Nucleotide Sequence Data. Ph. D. thesis, University of Colorado.

[Olsen 1987] Olsen, G. J., Earliest phylogenetic branchings: Comparing rRNA-based evolutionary trees inferred with various techniques. Cold Spring Harbor Symposia on Quantitative Biology LII, 825-837.

[Pfluger, Overbeek, and Winker 1990] Pfluger, N., Overbeck, R., and Winker, S., Argonne National Laboratory, unpublished information.

[Sankoff and Kruskal 1983] Sankoff, D., and Kruskal, J., Time Warps, Siring Edits, and Macromolecules: The Theory and Practice of Sequence Comparison. Addison-Wesley, Reading, Massachusetts.

[Stern, Weiser, and Noller 1988] Stern, S., Weiser, B., and Noller, H. F., A model for the threedimensional folding of 16 S ribosomal RNA. Journal of Molecular Biology 204, 447-481.

[Stiegler et al. 1981] Stiegler, P., Carbon, P., Zuker, M., Ebel, M.-P., and Ehresmann, C., Structural organization of the $16 \mathrm{~S}$ ribosomal RNA from $E$. coli. Topography and secondary structure. Nucleic Acids Research 9.2153-2172.

[Thanaraj et al. 1989] Thanaraj, T. A., Kolaskar, A. S., and Pandit, M. W., An extension of the graph theoretical approach to predict the secondary structure of large RNAs: The complex of $16 S$ and 23S rRNAs from E. coli as a case sthdy. Comp. Appl. Biosci. 5, 211-218.

[Watson et al. 1987] Watson, J. D., Hopkins, N. H., Roberts, J. W., Steitz, J. A., and Weiner, A. M., Molecular Biology of the Gene. 4th Edition, Benjamin/Cummings, Menlo Park, California.

[Woese and Gutell 1989] Woese, C. R., and Gutell, R. R., Evidence for several higher-order structural elements in ribosomal RNA. Proc. Natl. Acad. Sci. (USA) 86, 3119-3122.

[Woese et al. 1983] Woese, C. R.. Gutell, R. R., Gupta, R., and Noller, H. F., Detailed analysis of the higher order structure of 16S-like ribosonial ribunucleic acids. Microbiological Reviews 47, 621-669.

[Woese et al. 1980] Woese, C. R., Magrum, L. J., Gupta, R., Siegel, R. B., Stahl, D. A., Kop, J., Crawford, N., Brosius, J., Gutell, R. R., Hogan, J. J., and Noller, H. F., Secondary structure model for bacterial $16 S$ ribosomal RNA: Phylogenetic, enzymatic, and chemical evidence. Nucleic Acids Research 6 (10), 22752293.

[Zuker 1989] Zuker, M., On finding all suboptimal foldings of an RNA molecule. Science 244, 48-52.

[Zuker and Stiegler 1981] Zuker, M., and Stiegler, P., Optimal computer folding of large RNA sequences using thermodynamics and auxiliary information. Nucleic Acids Research 9, 133-148. 